\title{
Active torque distribution strategy for electric vehicle independent axle drive
}

\author{
Artur Kopczyński ${ }^{1, *}$ \\ ${ }^{1}$ Warsaw University of Technology, Department of Multisource Propulsion Systems, ul. Narbutta 84, \\ 02-524 Warsaw, Poland
}

\begin{abstract}
Vehicles equipped with an electric independent axle drive have different properties compared to conventional vehicles. Distribution of driving or braking torque can be achieved by the proper control of the operation of electric machines without applying additional mechanisms. In this paper a method of active torque distribution between front and rear axles is presented. The method allows to use the maximum tyres adhesion and minimize slips. The results of simulation tests are presented, in which the active torque distribution with evenly torque distribution were compared.
\end{abstract}

\section{Introduction}

Electric cars, different in many respects compared to conventional vehicles powered by fossil fuels, are characterised by distinctive features widely discussed in all types of media sources nowadays. Some of them like obvious issues related to driving range [1-3], energy consumption [4, 5], charge or refuel time [6] or impact on the local environment [7] are most often repeated topics. However, there are also other aspects scrutinized more deeply by scientists. These include for example the possibility of energy recovery from the braking process $[8,9]$ or the fact that the electric drive is much more compact, which creates the possibility of greater diversity in the structure of the propulsion system. In an electric vehicle, the motors can for instance be placed in wheel hubs, or two machines can independently drive the front and rear axles of wheels $[10,11]$. The problem of the distribution of drive torques in multimotor electric powertrain is a normal consequence of the development of technology. In articles $[12,13]$, the issue of controlling the electric independent axle drive of the vehicle in the event of loss of adhesion by one of the wheels was analysed. However, relatively few research papers address the issue of how to distribute the drive torque between axles or wheels to minimize the risk of losing adhesion by tyres.

\section{Electric vehicle independent axle drive}

The electric vehicle independent front and rear axle drive scheme is shown in Figure 1. The vehicle is driven by electric motors (EM) and powered by an electrochemical battery (Bat). EM transmit the driving/braking torque to the wheels via the drive shafts and differential mechanism (X). The prototype Italian car P-Mob and commercially available Tesla Model S

* Corresponding author: artur.kopczynski@pw.edu.pl 
All-wheel Drive Dual Motor [14] are examples of such a construction. The correct operation of independent front and rear electric drive is handled by the Central Control Unit (CCU). CCU decides about actions such as torque distribution between axles or defines the mode of EMs operation (motor for accelerating and drive with constant speed and generator in case of regenerative braking). In Figure 1 the continuous (-) and dashed (-- - ) lines denote the mechanical and electrical connections, respectively. The arrows indicate the possible direction of the energy flow depending on the EM operating mode.

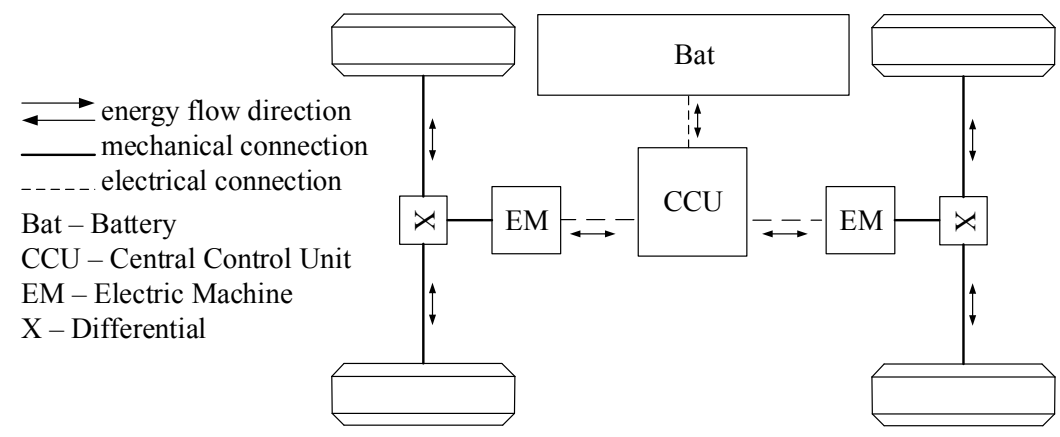

Fig. 1. Electric vehicle independent front rear axle drive.

\section{Modelling}

The preparation of appropriate simulation model is crucial to analyse vehicle's dynamic properties. One can refer to [15] in order to get acquainted with this topic. However, the most important elements that should be taken into consideration while creating such a simulation model adapted to the independent front and rear axle drive are presented below.

\subsection{Motion equation of vehicle equipped with independent axle drive}

The Equations (1-3) of motion of the vehicle equipped with independent axle drive are derived from the second law of Newton's dynamics.

$$
\begin{aligned}
d^{2} x / d t^{2} & =1 / m\left[\left\{\left[\tau_{F}+\tau_{R}\right] / r_{d}\right\}-F_{m r}\right] \\
\tau_{F} & =J_{F} d \omega_{F} / d t+F_{x F} r_{d} \\
\tau_{R} & =J_{R} d \omega_{R} / d t+F_{x R} r_{d}
\end{aligned}
$$

where: $x$ - means vehicle longitudinal displacement, $m$ - vehicle mass, $r_{d}$ - tyre dynamic radius, $F_{m r}$ - sum of motion resistance forces, $T F_{,}, T_{R}-$ front, rear wheels torque, $\mathrm{J}_{\mathrm{F}}, \mathrm{J}_{\mathrm{R}}-$ front and rear wheels moment of inertia, $\omega_{F}, \omega_{R}-$ front, rear wheels angular speed, $\mathrm{F}_{\mathrm{xF}}, \mathrm{F}_{\mathrm{xR}}-$ front, rear tyre longitudinal force.

\subsection{Tyre}

A basic classic tyre model was adopted, taking into account only progressive (longitudinal) movement. The Equation (4) describes tyre longitudinal slip $s_{x}$ during vehicle acceleration 
due to tyre elasticity [16]. Whereas, the slip during the vehicle braking is expressed by the Equation (5).

$$
\begin{gathered}
s_{x}=\left[r_{d} \omega-v_{x}\right] / r_{d} \omega \\
s_{x}=\left[v_{x}-r_{d} \omega\right] / v_{x}
\end{gathered}
$$

When driving or braking torque is applied to the wheels, tyre deformation occurs. In such a situation tyre wheel has less ability to transfer power. An example of the characteristics of the coefficient of adhesion $\mu_{x}$ as a function of tyre slip $s_{x}$ is presented in Figure 2.

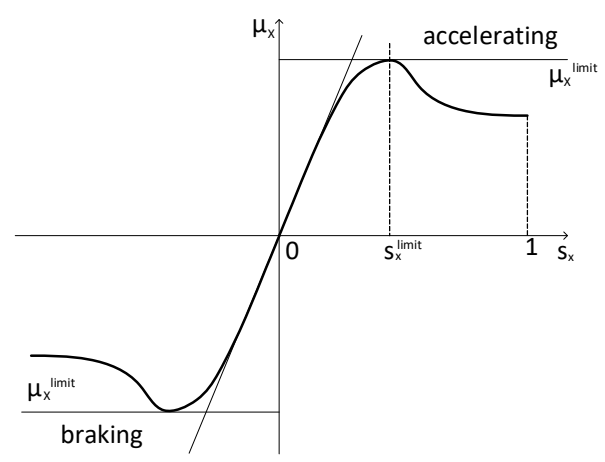

Fig. 2. Tyre adhesion coefficient characteristic.

Taking into account the slip, the force transmitted by the tyre is expressed by Equation (6).

$$
F_{x}=\mu_{x} F_{z}
$$

where: $F_{X}$ - means tyre longitudinal force and $F_{z}$ - tyre vertical load. In order to analyse the motion of the vehicle cornering, it is necessary to extend the model with lateral deformation of the tyre [16].

\subsection{Wheel vertical load caused by pitch}

The method of determination of vertical load caused by pitch (during accelerating or braking) was presented in the work [8]. Forces of vertical load of a given axle during vehicle accelerating are expressed by Equations (7) and (8), where $a$ - means vehicle acceleration or deceleration, $h$ - height of COG (Centre of Gravity) from ground and $L$ - wheelbase.

$$
\begin{aligned}
& F_{z f}=\left\{m\left[g l_{r}-a h\right]\right\} / L \\
& F_{z r}=\left\{m\left[g l_{f}+a h\right]\right\} / L
\end{aligned}
$$

In case of vehicle braking the forces $F_{z f}$ and $F_{z r}$ are defined by similar Equations to $(7,8)$ but the signs in numerators are opposite.

\section{Torque distribution strategy}

In conventional cars, a differential mechanism with two degrees of freedom is used to compensate the difference in wheels speed. In $4 \times 4$ vehicles, this differential mechanism 
is also located between the front and rear axles. The relation between speeds of the differential mechanism is described by Equation (9).

$$
\left[\omega_{F}+\omega_{R}\right] / 2=\omega_{\text {engine }}
$$

While the torque on each exit shafts $T_{1}, T_{2}$ from differential mechanism is described by Equation (10) and (11) [16].

$$
\begin{aligned}
& T_{1}=0.5 T_{0}+0.5 T_{f} \\
& T_{2}=0.5 T_{0}+0.5 T f
\end{aligned}
$$

If the value of internal friction torque $T f$ is small (as it is for most common differential mechanisms), then the torque is distributed evenly.

In the case of independent front and rear electric powertrain, any torque on both motors can be set (limited by ME parameters). Equation (12) describes the active torque distribution method between the front $T F$ and the rear $T_{R}$, based on the maximum usage of tyre adhesion.

$$
T F / T_{R}=\left[g l_{r}-a h\right] /\left[g l_{r}+a h\right]
$$

The Equation (12) results directly from the relation of vertical loads occurring on both wheel axles of the vehicle, which is described by Equations (7) and (8).

Figure 3 shows the percentage torque distribution during acceleration, while Figure 4 refers to the braking of the vehicle. Both graphs present results for a vehicle with the parameters given in Table 1 . The acceleration plot has been limited to $a=4 \mathrm{~m} / \mathrm{s}^{2}$, because it is the highest acceleration that the analysed vehicle can reach. For both cases (acceleration/braking) the initial difference (for $a=0 \mathrm{~m} / \mathrm{s}^{2}$ ) results from the position of the centre of gravity. As the acceleration / deceleration value changes, the relation between torques on front and rear wheels also differentiates.

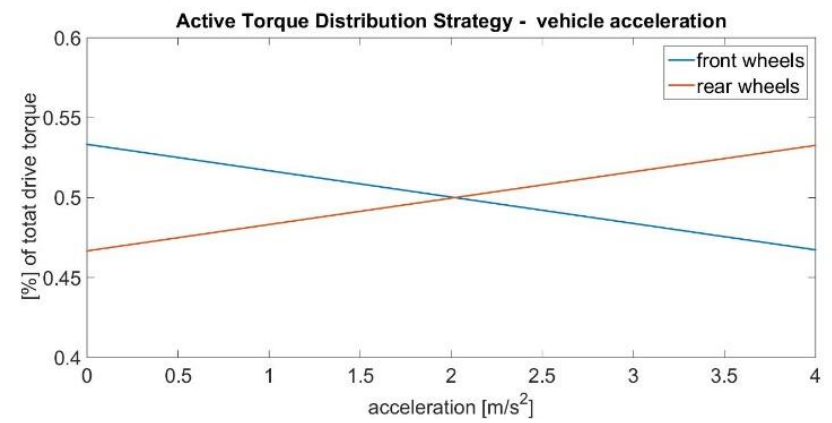

Fig. 3. Active torque distribution during vehicle accelerating. 


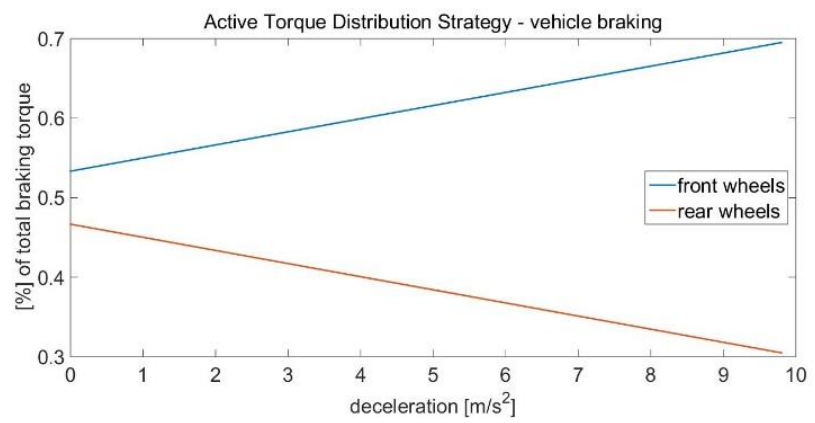

Fig. 4. Active torque distribution during vehicle braking.

\section{Simulation study}

Simulation tests were carried out for a small electric vehicle, the parameters of which were defined in Table 1.

Table 1. Input data.

\begin{tabular}{|c|c|}
\hline Vehicle Parameter & Value \\
\hline Mass & $\mathrm{m}=500 \mathrm{~kg}$ \\
\hline Frontal area & $\mathrm{A}=1.35 \mathrm{~m}^{2}$ \\
\hline Drag coefficient & $\mathrm{Cd}=0.45$ \\
\hline Rolling resistance coefficient & $\mathrm{ft}=0.01$ \\
\hline Tyre dynamic radius & $\mathrm{rd}=0.27 \mathrm{~m}$ \\
\hline Front axle to COG & $\mathrm{lf}=1.0 \mathrm{~m}$ \\
\hline Rear axle to COG & $\mathrm{lr}=1.5 \mathrm{~m}$ \\
\hline Centre of gravity height & $\mathrm{h}=0.34 \mathrm{~m}$ \\
\hline Rotor inertia & $\mathrm{J}=0.128 \mathrm{kgm}^{2}$ \\
\hline Wheel inertia & $\mathrm{Jk}=0.7 \mathrm{kgm}^{2}$ \\
\hline
\end{tabular}

Acceleration of the vehicle on a straight road was simulated. The results of these tests are presented in Figures 5-10. In the graphs in Figures 5 and 6 the waveforms of vehicle's velocity and acceleration for the two analysed strategies were compared, i.e. evenly and active torque distribution. In both cases, the waveforms are very similar to each other, which allows a good comparison of subsequent parameters.

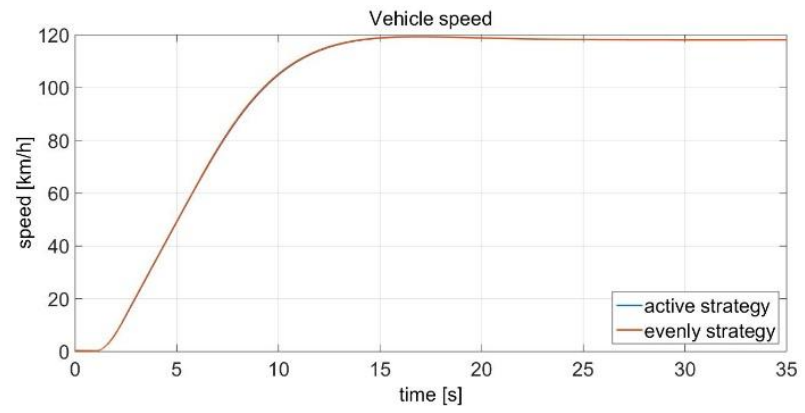


Fig. 5. Vehicle longitudinal speed in tested scenario.

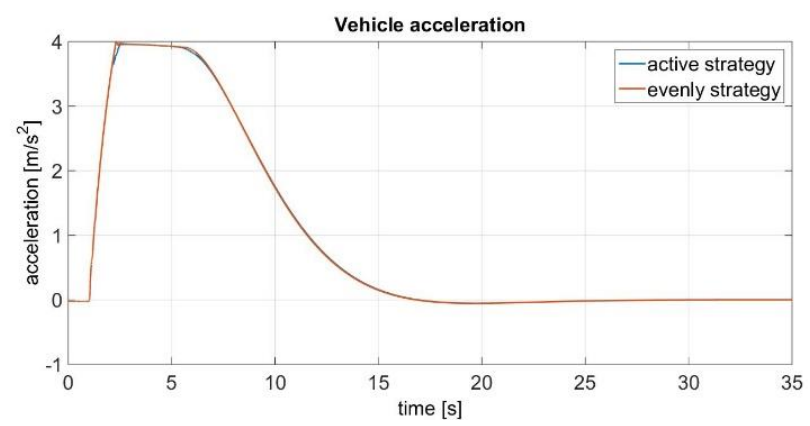

Fig. 6. Vehicle longitudinal acceleration in tested scenario.

The torque on wheels waveforms on the front and rear axle for evenly strategy and the waveforms of tyres slips are shown in Figures 7 and Figure 8, respectively. The torque graph for both axles is almost identical, which is in line with the assumptions of this strategy. In the case of slips, there is a significant difference in the time range when the vehicle achieves the greatest acceleration. The values of slip on the front axle are higher because this axis is less loaded during acceleration (according to Equations 7 and 8 ) and their ability to transfer torque is lower.

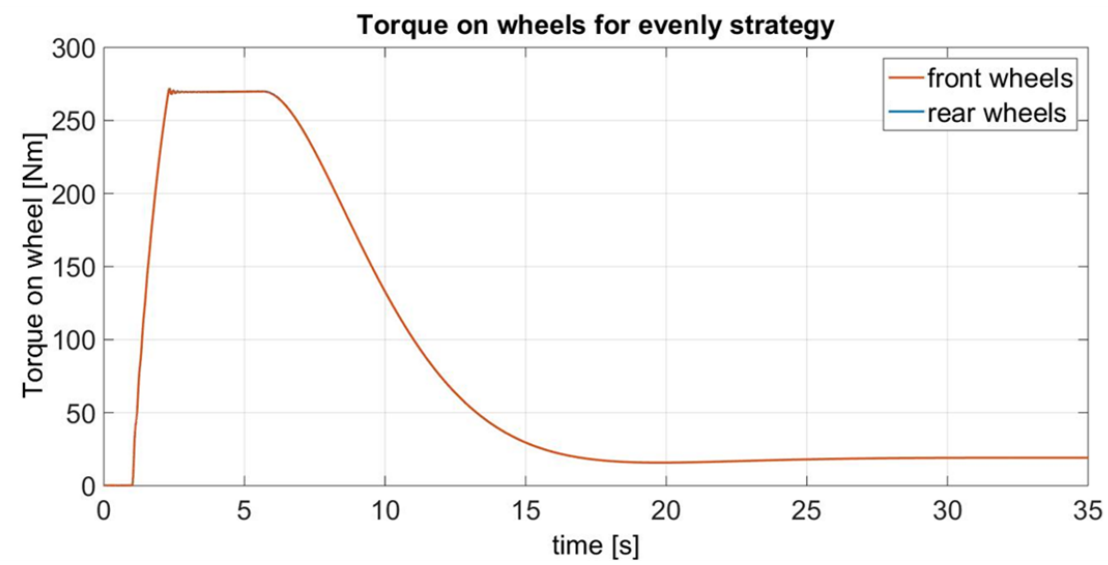

Fig. 7. Front and rear wheels torques for evenly torque distribution strategy.

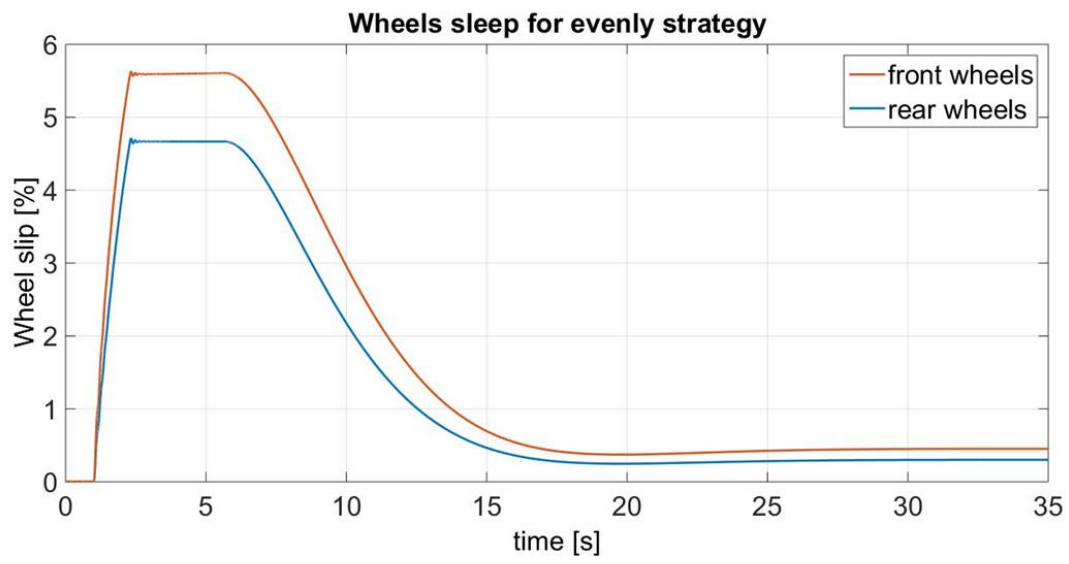


Fig. 8. Front and rear wheels tyres slip for evenly torque distribution strategy.

The simulation results of active torque distribution, are shown in Figures 9 and 10. Greater drive torque was delivered to the rear wheels of the vehicle, because these wheels are loaded during acceleration, and thus tyres have better adhesion with ground. The torque of the front axle was proportionally lower. Due to active distribution, slips on both axes adopted similar values which means that the vehicle has a greater stability margin. Potential loss of stability of vehicle movement occurs at the moment of maximum use of adhesion by all wheels. With this method, all wheels approach their adhesion limits before losing adhesion on one of them. As a consequence, the risk of losing adhesion by one of the wheels is lower than in the evenly distribution strategy.

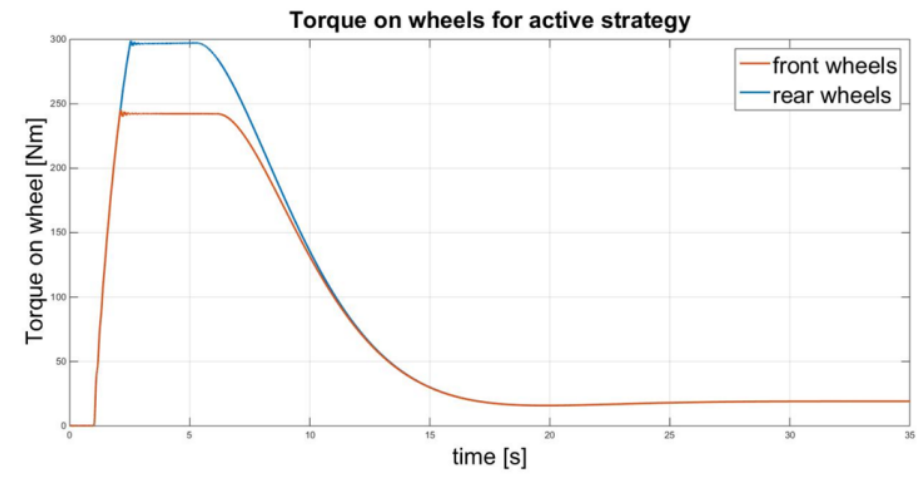

Fig. 9. Front and rear wheels torques for active torque distribution strategy.

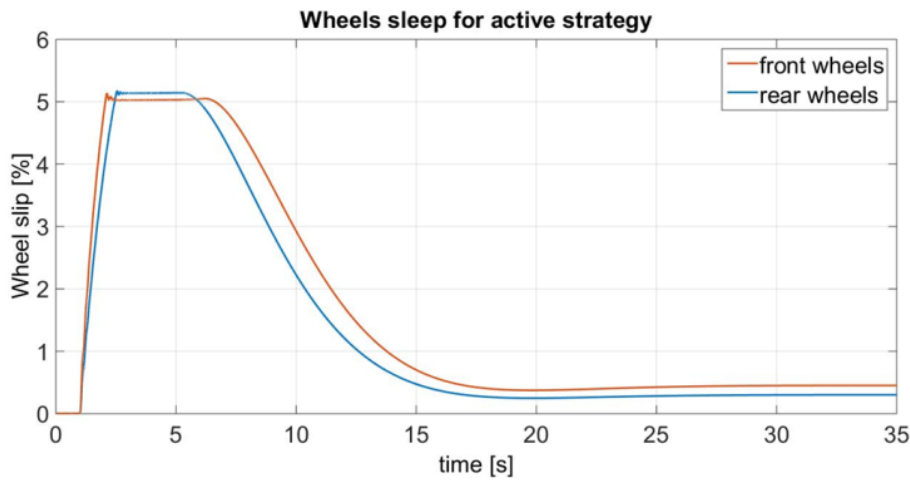

Fig. 10. Front and rear wheels tyres slip for active torque distribution strategy.

\section{Conclusion}

The presented method of active torque distribution allows to better use of tyre adhesion, and thus positively affects vehicle stability. In comparison to the evenly distribution strategy, slips on all wheels reach similar values. For a vehicle with a higher EM power, it would be possible to approach the limit values of wheel slip. Therefore, the differences in the indicated parameters (discussed in chapter 5) would be even greater, and of course in favour of the active method. The position of COG has also a significant influence on the differences in the results. The higher COG is located from the ground and the closer it is to one of the wheel axles, the advantage of the active distribution strategy will be greater. A slight drawback of the indicated method is the operation of torque distribution algorithm depending on vehicle's momentary longitudinal acceleration. In a real car, it is possible to define the function of the 
dependence of the current work parameters of EMs and the position of the accelerator pedal and determine the theoretical relations on the acceleration of the vehicle. On the other hand, in modern vehicles equipped with ESP (Electronic Stability Program) systems, acceleration sensors in all three directions are widely used, so this should not be a problem. Another weak point of the indicated method as well as other such systems is sensitivity to the position of COG, which depending on whether only the driver, or e.g. 5 passengers and luggage are travelling, creates a significant difference. However, taking into account all the abovementioned aspects and the results of simulation tests, the presented method of active torque distribution is significantly better than the evenly distribution method.

\section{References}

1. A. Hajduga, Proceedings of $23^{\text {rd }}$ International Conference on Methods \& Models in Automation \& Robotics (MMAR), 843-848 (2018)

2. A. Kopczyński, P. Krawczyk, J. Lasocki, E3S Web of Conferences 44, 00073 (2018)

3. A. Hajduga, Proceedings of $23^{\text {rd }}$ International Conference on Methods \& Models in Automation \& Robotics (MMAR), 622-627 (2018)

4. A. Szumanowski, Y. Chang, Z. Liu, P. Krawczyk, Int. J. Vehicle Des. 76, 1-19 (2018)

5. A. Kopczyński, P. Piórkowski, P. Roszczyk, IOP Conf. Ser. Mater. Sci. Eng. 421, 022016 (2018)

6. A. Kopczyński, Z. Liu, P. Krawczyk, E3S Web of Conferences 44, 00074 (2018)

7. Z. Chłopek, J. Lasocki, P. Wójcik, A.J. Badyda, Int. J. Green Energy 15, 773-779 (2018)

8. A. Kopczyński, Logistyka: czasopismo dla profesjonalistów 3, 2269 (2015)

9. N. Mutoh, IEEE Trans. Ind. Electron. 59, 10 (2012)

10. N. Mutoh, K. Takita, Conference Record of the 2004 IEEE Industry Applications Conference, $39^{\text {th }}$ IAS Annual Meeting, 8245720 (2004)

11. L. Wei, H. Hongwen, Y. P. Yang, C. P. Lo, Control Eng. Pract. 16, 1285-1292 (2008)

12. H. Hongwen, P. Jiankun, X. Rui, F. Hao, Energies 7, 3748-3763 (2014)

13. W. Liu, H. He, J. Peng, Math. Probl. Eng. 2013, 408965 (2013)

14. https://www.tesla.com/sites/default/files/tesla-model-s.pdf

15. A. Kopczyński, P. Krawczyk, Proceedings of the Institute of Vehicles 112, 49 (2017)

16. D. Schramm, M. Hiller, Vehicle Dynamics Modeling and Simulation (Springer, 2014) 